\title{
Suspected Neuro-invasive Powassan Virus Infection in a Pediatric Patient
}

\author{
Trever M. Koester, BS; Priyanka Timothy, MD; Jennifer K. Meece, PhD; \\ Rebecca A. Osborn, MPH; and Holly M. Frost, MD
}

\begin{abstract}
Powassan virus lineage II (POWV), also known as deer tick virus, is an emerging tick-borne pathogen transmitted by Ixodes scapularis, the natural vector for the organisms that causes Lyme disease, babesiosis, and anaplasmosis. POWV is the only tick-borne flavivirus in North America known to cause disease in humans. We present a suspected pediatric case of POWV infection in northern Wisconsin.
\end{abstract}

Keywords: Arbovirus; Encephalitis; Pediatric; Powassan virus; Tick-borne disease,

$\mathrm{P}$ owassan virus lineage II (POWV), also known as deer tick virus, is a genetically distinct member of the mammalian tick-borne encephalitis virus group. It is an emerging tick-borne pathogen transmitted by Ixodes scapularis, the natural vector for the organisms that cause Lyme disease, babesiosis, and anaplasmosis. POWV is the only tick-borne flavivirus in North America known to cause disease in humans. In the United States, human incidence of POWV has increased in the last 15 years. ${ }^{1}$ We present a suspected pediatric case of POWV infection in northern Wisconsin.

\section{Case Report}

In August 2017, a previously healthy, Caucasian female, aged 8 years, presented to a clinic in Wisconsin with a one-day history of headache, photophobia, fever to $101^{\circ} \mathrm{F}$, lethargy, poor oral intake, diffuse abdominal pain, and vomiting. Three days prior, the patient was camping and had exposure to lake water, wooded areas, mosquitoes, and other insects. No tick bite was noted. The patient reportedly lived next door to an uncle who was recently diagnosed with Rocky Mountain Spotted Fever (RMSF). The patient had no history of travel outside of Wisconsin within the past 30 days. On arrival to the clinic, her physical examination was notable for stiff neck, but was otherwise non-focal. Laboratory studies at that time included a negative rapid Group A Streptococcus antigen test, negative urinalysis, complete blood cell count with leukocytosis (white blood cells of $26.3 \times 10^{3} / \mathrm{L}$; ref $4.5-13.5 \times$ $\left.10^{3} / \mathrm{L}\right)$, elevated C-reactive protein $(2.8 \mathrm{mg} / \mathrm{dL}$; ref $<1.0 \mathrm{mg} /$ dL) and mild hyponatremia (133 mEq/L; ref 133-144). Given her persistent vomiting, an abdominal ultrasound to evaluate for appendicitis was completed and was normal. Due to her symptom severity and neck stiffness, the patient was transferred to Marshfield Medical Center in Marshfield, Wisconsin for further management.

On arrival to our facility, the patient's diffuse headache persisted, and she admitted to jaw pain but specifically denied neck pain. She complained of abdominal pain, which she attributed to hunger along with dysuria and nocturnal enuresis. She was afebrile with vital signs within normal limits. Computed tomography scan without contrast of the brain was completed and was normal. Reverse transcriptase polymerase chain reaction (RT-PCR) in whole blood was negative for Anaplasma phagocytophilum, Babesia microti, and Ehrlichia chaffeensis. Two-tier Lyme serology was negative. Lumbar puncture was performed and showed red blood cells at 1 cells $/ \mu \mathrm{L}$ (ref 0 cells $/ \mu \mathrm{L}$ ) and elevated white blood cells at 88 cells $/ \mu \mathrm{L}$ (ref 0-5 cells $/ \mu \mathrm{L}$ ) with $0 \%$ blasts, $23 \%$ neutrophils, $66 \%$ lymphocytes, and $11 \%$ monocytes. Total protein was $35 \mathrm{mg} / \mathrm{dL}$
Corresponding Author: Trever M Koester, BS, Medical Student, Harvard Medical School, 25 Shattuck Street, Boston, MA 02II5, Tel: (715) 222-4566, Email: trever_ koester@hms.harvard.edu
Received: June 17, 2019

Ist Revision: October 13,2019

2nd Revision: December 20, 2019

Accepted: January 2, 2020

doi: $10.3121 / \mathrm{cmr} \cdot 2020.1512$ 
(ref $15-45 \mathrm{mg} / \mathrm{dL}$ ), and glucose was $66 \mathrm{mg} / \mathrm{dL}$ (ref 40-70 mg/ $\mathrm{dL})$. Ceftriaxone was started (50 mg/kg every 12 hours) for possible Lyme meningitis, and doxycycline was started $(2 \mathrm{mg} /$ kg every 12 hours) empirically for treatment of possible RMSF. Cerebrospinal fluid (CSF) was sent for culture as well as Lyme, Herpes Simplex Virus (HSV)-1, HSV-2, and Varicella Zoster Virus (VZV) PCR. An arbovirus IgM capture enzyme-linked immunosorbent assay (MAC-ELISA) panel was ordered on serum and CSF through the Wisconsin Division of Public Health.

On the second day of hospitalization, Lyme, HSV-1, HSV-2, and VZV CSF PCR returned negative, and CSF cultures had no growth. Ceftriaxone was discontinued. Throat culture showed light growth of Group A Streptococcus, and the patient was started on amoxicillin, which was continued for a total of 10 days. Despite treatment, the patient's symptoms persisted with fever to a maximum temperature of $102.2^{\circ} \mathrm{F}$, headache, and nausea. On the fifth night of hospitalization, the patient was playing a board game and developed periods of confusion with inability to understand rows, columns, and letters. On examination, a tremor was noted bilaterally in her hands, particularly with finger to nose testing. The tremor was noticeably worse on the left side. She also experienced urinary urgency and progressive ataxia with inability to ambulate without assistance. As the night progressed, she developed expressive aphasia. Neurology was consulted and recommended magnetic resonance imaging (MRI) of the brain and repeat CSF studies.

The brain MRI with and without contrast was normal. Repeat lumbar puncture showed RBCs at 1 cell $/ \mu \mathrm{L}(\mathrm{ref} 0$ cell $/ \mu \mathrm{L})$ and white blood cells at 39 cells $/ \mu \mathrm{L}$ ( ref $0-5$ cells $/ \mu \mathrm{L}$ ) with $84 \%$ lymphocytes, $11 \%$ monocytes, $0 \%$ blasts, and $5 \%$ neutrophils. Total protein was $58 \mathrm{mg} / \mathrm{dL}(15-45 \mathrm{mg} / \mathrm{dL})$, and glucose was $54 \mathrm{mg} / \mathrm{dL}$ (ref 40-70 mg/dL). Given concern for worsening meningoencephalitis, she was started on methylprednisolone $250 \mathrm{mg}$ IV $(8.4 \mathrm{mg} / \mathrm{kg})$ daily. On hospital day 8 the patient began to show improvement, and her neurologic examination returned to baseline. Steroids were decreased on day 9 of hospitalization to half the dose of methylprednisolone without worsening of neurologic symptoms and were subsequently discontinued. The patient was discharged on hospital day 10 with amoxicillin for Group A Streptococcus pharyngitis and doxycycline for concerns of RMSF.

After discharge, RMSF IgM returned negative. Arbovirus MAC-ELISA IgM and PRNT $_{90}$ results are shown in the Table 1. The arbovirus MAC-ELISA IgM panel in serum (collected on day 4 of illness) was positive for POWV and Jamestown Canyon virus (JCV). Serum IgM for West Nile virus (WNV), St. Louis encephalitis virus (SLEV), eastern equine encephalitis virus (EEEV), and La Crosse virus (LACV) were negative. Serum plaque reduction neutralization tests $\left(\mathrm{PRNT}_{90}\right)$ were positive for POWV (titer 1:40), JCV (titer $1: 640)$, and LACV (1:20), providing evidence of infections with POWV and JCV. CSF IgM from the initial lumbar puncture was positive for POWV and JCV, and negative for LACV, WNV, SLEV, and EEEV. PRNT 90 for JCV and LACV were completed on the initial (day 4) CSF sample and were negative (titer $<1: 2$ ); insufficient quantity of CSF was available to run POWV PRNT 90 in the initial CSF specimen. CSF IgM from the second lumbar puncture (collected on day 8 of illness) was positive for JCV, POWV, equivocal for LACV, and negative for WNV, SLEV, and EEEV. $\mathrm{PRNT}_{90}$ for POWV was completed on the day $8 \mathrm{CSF}$ specimen and was positive (titer 1:4); insufficient quantity of CSF was available to run JCV and LAC PRNT $_{90}$ in the day 8 specimen. A convalescent serum was requested but was unable to be obtained, as the patient was lost to follow-up and no residual specimen was available to complete metagenomic sequencing. While serum antibody testing suggested recent infections with both POWV and JCV, neutralizing antibodies on $\mathrm{PRNT}_{90}$ against $\mathrm{POWV}$, but not JCV, were detected in CSF. The patient's meningoencephalitis was, therefore, thought to be most likely due to POWV.

\section{Discussion}

To date roughly 100 cases of neuro-invasive POWV disease have been reported in the United States, with a steady increase in reported cases since 2006. ${ }^{1}$ Historically, testing has been limited to patients with neuro-invasive disease and, thus, both the breadth of disease and the incidence of infection are likely much larger than what has been reported. The sero-prevalance of POWV in North America ranges from $0.5 \%-4 \%$ in asymptomatic patients, with seroprevalence as high as $9.5 \%$ in patients presenting for Lyme disease testing in an endemic region in the Midwest. ${ }^{2}$ Up to $16 \%$ of I. scapularis ticks in some regions carry the virus, which can be transmitted in as little as 15 minutes after attachment. ${ }^{3}$ The rapid expansion of the I. scapularis territory, combined with increased awareness and testing capabilities, likely explain the increasing incidence. ${ }^{1}$

POWV is detected with an IgM MAC-ELISA or an IgM immunofluorescence antibody (IFA) assay. Cases are confirmed by $\mathrm{PRNT}_{90}$, detection of virus-specific nucleic acids (PCR), isolation in culture, or a $>4$-fold increase in antibody titers from paired acute and convalescent sera or $\mathrm{CSF}^{4}$ It is important to note that while PCR testing of CSF for tick-borne diseases and arboviruses can confirm a diagnosis, a negative result cannot exclude a diagnosis. As with most serologic assays, POWV IgM assays show considerable cross-reactivity with other viruses, and IgM may not be present in the first few days of infection. Thus, both false positive and negative results are common and serologic assays must be interpreted with caution. $\mathrm{PRNT}_{90}$ in acute and convalescent samples and/or direct detection of nucleic acids via PCR or metagenomic sequencing are necessary to confirm the diagnosis. ${ }^{4,5}$ In this case, serum tests detected antibodies against both JCV and POWV. Since illnesses caused by POWV and JCV are both rare, and these two viruses are transmitted by different vectors, concurrent infection is unlikely, and the presence of JCV serum IgM most likely indicates prior JCV infection with persistent elevated 
IgM. Persistence of detectable IgM from prior infection with JCV infection may occur, as it has been documented to occur after infection with other arboviruses. In comparison, the patient's CSF specimens were also IgM positive for POWV and JCV with equivocal results for LACV. However, subsequent CSF PRNT 90 was negative for JCV and LAC and positive for $\mathrm{POWV}$, indicating that neuro-invasive infection was most likely secondary to POWV. It is important to note that IgM ELISAs and PRNT ${ }_{90}$ S were completed on different CSF specimens taken 4 days apart. Thus, though unlikely, concurrent neuro-invasive infection with JCV cannot be completely excluded, as the patient may not have seroconverted when the $\mathrm{PRNT}_{90}$ was completed.

The POWV case fatality rate in adults is $10 \%-15 \%$, with $50 \%$ of patients experiencing neurologic sequelae. ${ }^{1}$ Though up to $17 \%$ of POWV cases occur in children, only two prior pediatric case reports have been described in the literature. ${ }^{6,7}$ Thus, optimal management of POWV encephalitis and clinical outcomes for children are poorly understood. Prior pediatric case studies have reported using methylprednisolone or anticonvulsant therapy. In adult patients with severe disease, intravenous immunoglobulin, ribavirin/interferon, and steroids have been utilized with success. Care for encephalitis, including for other arboviral diseases such as WNV, is largely supportive. Steroids and intravenous immunoglobulin for treatment of pediatric encephalitis have not been shown to be efficacious, though studies have been severely limited by sample size. Given the severe worsening in clinical symptoms in our patient, methylprednisolone was given and resulted in significant improvement.

There was concern for RMSF in this patient, though testing was ultimately negative. RMSF, caused by Rickettsia

Table 1. Arbovirus diagnostic test results from serum and cerebral spinal fluid on day 4 and 8 of illness.

\begin{tabular}{|c|c|c|}
\hline & Day 4 & Day 8 \\
\hline \multicolumn{3}{|l|}{ Serum } \\
\hline \multicolumn{3}{|l|}{ MAC-ELISA IgM ${ }^{1}$} \\
\hline Powassan virus & positive & -- \\
\hline Jamestown Canyon virus & positive & -- \\
\hline West Nile virus & negative & -- \\
\hline St. Louis encephalitis virus & negative & -- \\
\hline Eastern equine encephalitis virus & negative & -- \\
\hline La Crosse virus & negative & -- \\
\hline \multicolumn{3}{|l|}{$\mathrm{PRNT}_{90}{ }^{2}$} \\
\hline Powassan virus & $1: 40$ & -- \\
\hline Jamestown Canyon virus & $1: 640$ & -- \\
\hline La Crosse virus & $1: 20$ & -- \\
\hline \multicolumn{3}{|l|}{ Cerebral Spinal Fluid } \\
\hline \multicolumn{3}{|l|}{ MAC-ELISA IgM } \\
\hline Powassan virus & positive & positive \\
\hline Jamestown Canyon virus & positive & positive \\
\hline West Nile virus & negative & negative \\
\hline St. Louis encephalitis virus & negative & negative \\
\hline Eastern equine encephalitis virus & negative & negative \\
\hline La Crosse virus & negative & equivocal \\
\hline \multicolumn{3}{|l|}{$\mathrm{PRNT}_{90}^{2}$} \\
\hline Powassan virus & -- & $1: 4$ \\
\hline Jamestown Canyon virus & $<1: 2$ & -- \\
\hline La Crosse virus & $<1: 2$ & -- \\
\hline \multicolumn{3}{|c|}{$\begin{array}{l}{ }^{1} \text { IgM capture enzyme-linked immunosorbent assay (MAC-ELISA) was completed at Wisconsin State Laboratory of Hygiene. } \\
\text { ELISA measures optical density of patient serum dilution with viral antigen }(P) / \text { mean optical density of normal human } \\
\text { sera }(N) \text {. Result is positive if } P / N \geq 3.0 \text {. }\end{array}$} \\
\hline
\end{tabular}


rickettsii, is primarily transmitted by Dermacentor variabilis. RMSF is an uncommon pathogen in Wisconsin, with most cases being acquired from travel. However, the incidence of regionally-acquired RMSF has been increasing, and Wisconsin reported its first death from regionally-acquired RMSF in July 2018. Thus, given the high fatality rate of RMSF and deteriorating status of our patient, we believed it was appropriate to initiate treatment in this patient while awaiting test results.

In conclusion, POWV is an emerging tick-borne pathogen capable of causing neuro-invasive disease. Clinicians in Lyme endemic regions should maintain a high degree of suspicion for POWV given the high mortality and long-term morbidity. If POWV is suspected, POWV testing should be specifically requested, as many state arbovirus antibody panels do not routinely include this pathogen. It remains to be determined if viral detection changes clinical management. The optimal management remains to be determined but, similar to other arboviruses, is likely supportive care.

\section{Acknowledgements}

We would like to thank the Marshfield Clinic Research Institute summer research internship program for their support for this project.

\section{References}

1. Krow-Lucal ER, Lindsey NP, Fischer M, Hills SL. Powassan Virus Disease in the United States, 2006-2016. Vector Borne Zoonotic Dis. 2018;18(6):286-290.

2. Frost HM, Schotthoefer AM, Thomm AM, et al. Serologic Evidence of Powassan Virus Infection in Patients with Suspected Lyme Disease. Emerg Infect Dis. 2017;23(8):13841388.

3. Knox KK, Thomm AM, Harrington YA, Ketter E, Patitucci JM, Carrigan DR. Powassan/Deer Tick Virus and Borrelia Burgdorferi Infection in Wisconsin Tick Populations. Vector Borne Zoonotic Dis. 2017;17(7):463-466.

4. Centers for Disease Control and Prevention. Powassan Virus. Clinical Evaluation \& Disease. Available at: http://www.cdc.gov/ powassan/clinicallabeval.html. Accessed August 20, 2018.

5. Piantadosi A, Kanjilal S, Ganesh V, et al. Rapid Detection of Powassan Virus in a Patient With Encephalitis by Metagenomic Sequencing. Clin Infect Dis. 2018;66(5):789-792.

6. Hicar MD, Edwards K, Bloch K. Powassan virus infection presenting as acute disseminated encephalomyelitis in Tennessee. Pediatr Infect Dis J. 2011;30(1):86-88.

7. Tutolo JW, Staples JE, Sosa L, Bennett N. Notes from the Field: Powassan Virus Disease in an Infant - Connecticut, 2016. MMWR Morb Mortal Wkly Rep. 2017;66(15):408-409.

\section{Author Affiliations}

Trever M Koester, BS*; Priyanka Timothy, MD ; Jennifer K Meece, PhDf; Rebecca A Osborn, MPH§; and Holly M Frost, MD\|ף

*Department of Medicine, Harvard Medical School, Boston, Massachusetts, USA

†Department of Internal Medicine and Pediatrics, Marshfield Clinic

Health System, Marshfield, Wisconsin, USA

\#Integrated Research and Development Laboratory, Marshfield Clinic

Research Institute, Marshfield, Wisconsin, USA
$\S$ Wisconsin Department of Health Services, Madison, Wisconsin, USA $\|$ Department of Pediatrics, Denver Health and Hospital Authority, Denver, Colorado, USA

qDepartment of Pediatrics, University of Colorado School of Medicine, Aurora, Colorado, USA 